\title{
Dynamic Microtubule Ends Are Required for Growth Cone Turning to Avoid an Inhibitory Guidance Cue
}

\author{
Jean F. Challacombe, Diane M. Snow, and Paul C. Letourneau \\ Department of Cell Biology and Neuroanatomy, The University of Minnesota, Minneapolis, Minnesota 55455
}

Growth cone turning is an important mechanism for changing the direction of neurite elongation during development of the nervous system. Our previous study indicated that actin filament bundles at the leading margin direct the distal microtubular cytoskeleton as growth cones turn to avoid substratum-bound chondroitin sulfate proteoglycan. Here, we investigated the role of microtubule dynamics in growth cone turning by using low doses of vinblastine and taxol, treatments that reduce dynamic growth and shrinkage of microtubule ends. We used time-lapse phase-contrast videomicroscopy to observe embryonic chick dorsal root ganglion neuronal growth cones as they encountered a border between fibronectin and chondroitin sulfate proteoglycan in the presence and absence of $4 \mathrm{~nm}$ vinblastine or $7 \mathrm{~nm}$ taxol. Growth cones were fixed and immunocytochemically labeled to identify actin filaments and microtubules containing tyrosinated and detyrosinated $\alpha$-tubulin.

Our results show that after contact with substratum-bound chondroitin sulfate proteoglycan, vinblastine- and taxol-treated growth cones did not turn, as did controls; instead, they stopped or sidestepped. Even before drug-treated growth cones contacted a chondroitin sulfate proteoglycan border, they were narrower than controls, and the distal tyrosinated microtubules were less splayed and were closer to the leading edges of the growth cones. We conclude that the splayed dynamic distal ends of microtubules play a key role in the actin filament-mediated steering of growth cone microtubules to produce growth cone turning.

Key words: microtubule; growth cone; turning; actin filament; chondroitin sulfate proteoglycan; dynamic instability
Growth cones are the motile tips of elongating axons that guide growing axons to their targets during development of the nervous system. Growth cone navigation involves the detection and integration of extracellular signals, followed by a response that can include forward migration, retraction, branching, and turning. Detection of guidance cues is facilitated by protrusion and retraction of filopodia and lamellipodia from the peripheral region (P-domain) of the growth cone, which contains bundles and networks of actin filaments (AFs) (Letourneau and Ressler, 1983; Lewis and Bridgman, 1992). Axonal elongation depends on the advance of microtubules (MTs), which provide structural support and serve as tracks for axonal transport of membranous organelles. Stable MT bundles project from the axon into the central region (C-domain) of the growth cone, whereas dynamic MT ends splay apart and project into the actin-rich P-domain (Letourneau and Ressler, 1983; Gordon-Weeks, 1991; Challacombe et al., 1996).

Recent studies indicate that the advance of MTs into specific growth cone regions initiates responses to guidance cues, such as advance toward a target (Lin and Forscher, 1993), turning toward a positive cue (Bentley and O'Connor, 1994), and turning away from an unfavorable substratum (Tanaka and Kirschner, 1995) or

\footnotetext{
Received Nov. 7, 1996; revised Feb. 5, 1997; accepted Feb. 10, 1997.

This work was supported by National Institutes of Health Grants HD19950 (P.C.L.), F32-NS09971 (J.F.C.), and EY10545 (D.M.S.), and the Minnesota Medical Foundation. We thank Dr. G. Gallo for suggestions on this manuscript, Drs. A. I. Caplan and D. A. Carrino for their generous gift of chick limb bud CSPG, and Drs. J. C. Bulinski and G. G. Gundersen for providing antiserum against detyrosinated $\alpha$-tubulin.

Correspondence should be addressed to Jean F. Challacombe, Department of Cell Biology and Neuroanatomy, The University of Minnesota, 4-144 Jackson Hall, 321 Church Street SE, Minneapolis, MN 55455.

Dr. Snow's present address: Department of Anatomy and Neurobiology, The University of Kentucky, Lexington, KY 40536.

Copyright (C) 1997 Society for Neuroscience $\quad 0270-6474 / 97 / 173085-11 \$ 05.00 / 0$
}

inhibitory guidance cue (Challacombe et al., 1996). Interactions between AFs and MTs may regulate MT reorganization during these growth cone behaviors (Lin and Forscher, 1993; Bentley and O'Connor, 1994; Challacombe et al., 1996).

MTs are dynamic polymers, undergoing assembly and disassembly at their ends. These events are regulated by the gain and loss of a GTP cap from MT ends (Mitchison and Kirschner, 1984; Davis et al., 1994), the tubulin isotype composition (Panda et al., 1994), stabilization of MTs against loss of tubulin dimers (Bulinski and Gundersen, 1991), and several MT-associated proteins (MAPs) (for review, see Avila et al., 1994). In previous studies of MT dynamics, cells were treated with low concentrations of tubulin-binding drugs that suppress the dynamic instability of MT ends (Jordan et al., 1991, 1992, 1993; Zheng et al., 1993; Liao et al., 1995; Tanaka et al., 1995; Yu and Baas, 1995; Rochlin et al., 1996), showing that low concentrations of taxol, vinblastine, and nocodazole reduce MT dynamics and decrease the rate of neurite elongation (Letourneau and Ressler, 1984; Letourneau et al., 1986; Zheng et al., 1993; Tanaka et al., 1995; Yu and Baas, 1995; Rochlin et al., 1996).

Although these studies provide evidence that dynamic MTs are involved in neurite elongation, no one has examined their role in growth cone guidance. We used low concentrations of taxol and vinblastine to test the hypothesis that dynamic MT ends are required for the selective steering of MTs that is necessary for growth cone turning to avoid an inhibitory guidance cue, chondroitin sulfate proteoglycan (CSPG). Our results show that taxol and vinblastine partially reduced the rate of neurite elongation and completely inhibited growth cone turning. These findings indicate that splayed dynamic MT ends in the growth cone P-domain are necessary for the redirection of MTs and initiation of growth cone turning. 
Table 1. Rates of growth cone migration in the presence of low concentrations of taxol and vinblastine

Treatment

Growth cone migration rate (no. of growth cones)

DMSO control

$45.1 \pm 5.5(18)$

Pre-taxol

$39.9 \pm 4.2(26)$

7 nM taxol

$39.1 \pm 3.2(42)$

Taxol washout

$40.8 \pm 3.2(16)$

Pre-vinblastine

$43.9 \pm 3.6(30)$

4 nM vinblastine

$29.6 \pm 1.5(78)^{a}$

Vinblastine washout

$25.7 \pm 2.9(22)^{a}$

Growth cone migration rates were calculated by measuring the distances individual growth cones migrated (in micrometers) over a 15-60 min period. Rates were expressed as $\mu \mathrm{m} / \mathrm{hr} \pm$ SEM. Differences in growth cone migration rates among all groups were assessed by the Kruskal-Wallis test with post hoc multiple comparison. ${ }^{a}$ Significantly slower than pre-vinblastine rate $(p<0.05)$.

\section{MATERIALS AND METHODS}

Preparation of substrata. Heat-treated glass coverslips $(24 \times 30 \mathrm{~mm})$ were mounted over $22 \mathrm{~mm}$ holes drilled into the bottom of $50 \times 9 \mathrm{~mm}$ tissue culture dishes (Falcon Labware, Oxnard, CA) using aquarium sealant (Dow-Corning, Midland, MI). Coverslips were UV-sterilized for $30 \mathrm{~min}$, coated with $0.1 \mathrm{mg} / \mathrm{ml}$ poly-L-lysine for $1 \mathrm{hr}$ at $40^{\circ} \mathrm{C}$, rinsed, and coated with nitrocellulose as described previously (Lagenaur and Lemmon, 1987; Snow et al., 1990). Cellulose filter paper strips (Whatman No. 1) were soaked in a solution of chick limb bud CSPG (a generous gift from Drs. A. I. Caplan and D. A. Carrino, Case Western Reserve University, Cleveland, OH) (Carrino and Kaplan, 1985) containing 20\% rhodamine isothiocyanate, transferred to nitrocellulose-coated dishes (Snow et al., 1990), overlaid with 40-50 $\mu \mathrm{g} / \mathrm{ml}$ human plasma fibronectin (FN) (in PBS, pH 7.1; Life Technologies, Grand Island, NY), and incubated for $3-4 \mathrm{hr}$ at room temperature or overnight at $4^{\circ} \mathrm{C}$. Dishes were subsequently blocked with $5 \mathrm{mg} / \mathrm{ml}$ bovine serum albumin in PBS for $1 \mathrm{hr}$, then covered with culture medium and stored at $40^{\circ} \mathrm{C}$ in a humidified air chamber.

Cell culture. Dorsal root ganglia (DRG) were dissected from embryonic days 9-11 white Leghorn chicken embryos and cut into pieces to make explants. Explants were suspended in supplemented serum-free HEPESbuffered F14 medium (Letourneau et al., 1990). In some experiments, dissociated DRG sensory neurons were used (Letourneau et al., 1986). Dishes containing 20-25 explants or 20,000-30,000 sensory neurons were incubated at $40^{\circ} \mathrm{C}$ in a humidified air chamber for $16-48 \mathrm{hr}$.

Videomicroscopy. After incubation, a culture dish was placed on the stage of an inverted microscope (Diaphot, Nikon, Garden City, NY) under an air curtain incubator (ASI 400, Carl Zeiss, Thornwood, NY) at $40^{\circ} \mathrm{C}$. Growth cones were viewed by phase-contrast optics while rhodamine-labeled CSPG stripes were located under epi-illumination. Time-lapse phase-contrast images were acquired with a Newvicon video camera (Dage-MTI, Michigan City, IN) enhanced using Image 1 software
(Universal Imaging, West Chester, PA) and recorded with an optical disk recorder (Panasonic TQ-2026F or TQ-3038F, Panasonic Industrial, Secaucus, NJ).

Images of a microscope field containing several growth cones approaching a CSPG stripe were recorded once per minute until the growth cones were $100 \mu \mathrm{m}$ from the CSPG border. Then culture medium was replaced by medium containing either $7 \mathrm{nM}$ taxol (Natural Products Branch, National Cancer Institute, Bethesda, MD) or $4 \mathrm{~nm}$ vinblastine (Sigma, St. Louis, MO) diluted from stock solutions dissolved in dimethyl sulfoxide (DMSO) at concentrations of DMSO that did not exceed 3 $\mu \mathrm{l} / \mathrm{ml}$. A similar volume of DMSO alone was added to control cultures. Growth rates were calculated by measuring the distance that growth cones migrated (in micrometers) in successive recorded image frames using Image 1 software.

Fixation and immunocytochemistry. Cultures were fixed and extracted simultaneously for $10 \mathrm{~min}$ with PHEM buffer (60 mM PIPES, $25 \mathrm{~mm}$ HEPES, 10 mM EGTA, 2 mM $\mathrm{MgCl}_{2}$, pH 6.9) (Schliwa and van Blerkom, 1981; Letourneau and Ressler, 1983) containing $0.2 \%$ glutaraldehyde and $0.1 \%$ Triton $\mathrm{X}-100$, followed by one $15 \mathrm{~min}$ treatment with $1 \mathrm{mg} / \mathrm{ml}$ sodium borohydride in $\mathrm{Ca}^{2+}-\mathrm{Mg}^{2+}$-free PBS (CMF-PBS) and incubation for $15 \mathrm{~min}$ in a soaking solution (consisting of CMF-PBS containing $1 \%$ nonfat dry milk and $1 \mathrm{mg} / \mathrm{ml}$ sodium azide). MTs were labeled with rat monoclonal antibody YL $1 / 2$ directed against tyrosinated $\alpha$-tubulin (Kilmartin et al., 1982) (Sera-Labs) and rabbit polyclonal antibody against detyrosinated $\alpha$-tubulin (a generous gift from Drs. J. C. Bulinski and G. G. Gundersen, Columbia University) (Gundersen et al., 1984), used at 1:25 and 1:400 dilutions, respectively. AFs were labeled with rhodamine-conjugated phalloidin (Molecular Probes, Eugene, OR). Primary antibodies and phalloidin were diluted into soaking solution and applied for $45 \mathrm{~min}$. Dishes were rinsed with CMF-PBS to remove unbound antibodies, then soaked for $15 \mathrm{~min}$ in soaking solution. Fluorescein- and CY5-conjugated secondary antibodies (Jackson ImmunoResearch Laboratories, West Grove, PA), at 1:400 dilutions, were applied simultaneously for $45 \mathrm{~min}$. Dishes were rinsed, soaked, and coverslipped in a solution containing polyvinyl alcohol and glycerol.

Confocal microscopy. Immunofluorescence images were acquired with either a BioRad MRC 1000 (Challacombe et al., 1996) or BioRad MRC 1024 confocal laser scanning microscope (BioRad Microscience, Cambridge, MA), equipped with an Olympus Provis AX70 microscope and $60 \times$ objective, and krypton-argon laser (Brelje et al., 1993). To ensure that double- and triple-labeled images were in the greatest possible register, green, red, and/or far red fluorescence images were acquired with the same dichroic mirror. Digital images were reproduced using Adobe Photoshop version 3.0.

Quantitation of MT organization in growth cones. To quantitatively examine the organization of MTs in taxol- and vinblastine-treated growth cones, images of tyrosinated and detyrosinated $\alpha$-tubulin labeling were merged with images of phalloidin staining to view the positions of MTs within the growth cone relative to the leading edge and filopodia. Distances from the leading edge to the distal extents of both tyrosinated and detyrosinated MT staining, and widths of the MT labeling and growth cone were measured with BioRad MRC 1024 Laser Sharp software (version 2.1). For each condition, measurement data were pooled, and

Table 2. Effects of taxol and vinblastine on growth cone behavior at FN/CSPG borders

\begin{tabular}{|c|c|c|c|c|c|}
\hline Treatment & Stopped & Sidestepped & Turned & $\begin{array}{l}\text { Combinations of } \\
\text { stopped/ } \\
\text { retracted/ } \\
\text { sidestepped }\end{array}$ & Misc. $^{a}$ \\
\hline $\mathrm{DMSO}_{\text {control }}^{b}$ & $0 \%$ & $17 \%(4)$ & $61 \%(14)$ & $0 \%$ & $22 \%(5)^{c}$ \\
\hline Taxol (7 nM) & $11.1 \%(2)$ & $16.7 \%(3)$ & $0 \%$ & $66.7 \%(12)$ & $5.5 \%(1)^{d}$ \\
\hline Vinblastine (4 nM) & $23.8 \%(5)$ & $9.5 \%(2)$ & $0 \%$ & $57.2 \%(12)$ & $9.5 \%(2)^{e}$ \\
\hline
\end{tabular}

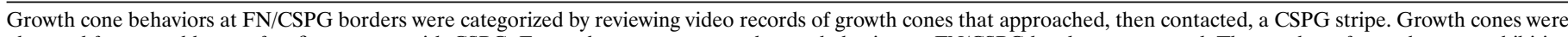

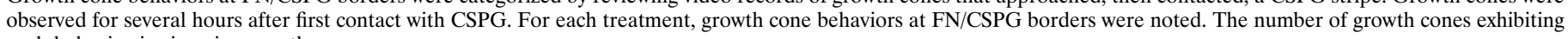
each behavior is given in parentheses.

${ }^{a}$ Misc., Miscellaneous.

${ }^{b}$ Data for DMSO controls from Challacombe et al. (1996).

${ }^{c}$ Five growth cones exhibited intermittent periods of stopping, sidestepping, and turning.

${ }^{d}$ One growth cone crossed the border after more than $4 \mathrm{hr}$ in the presence of $7 \mathrm{~nm}$ taxol.

${ }^{e}$ Two growth cones branched at the border, then both branches sidestepped. 

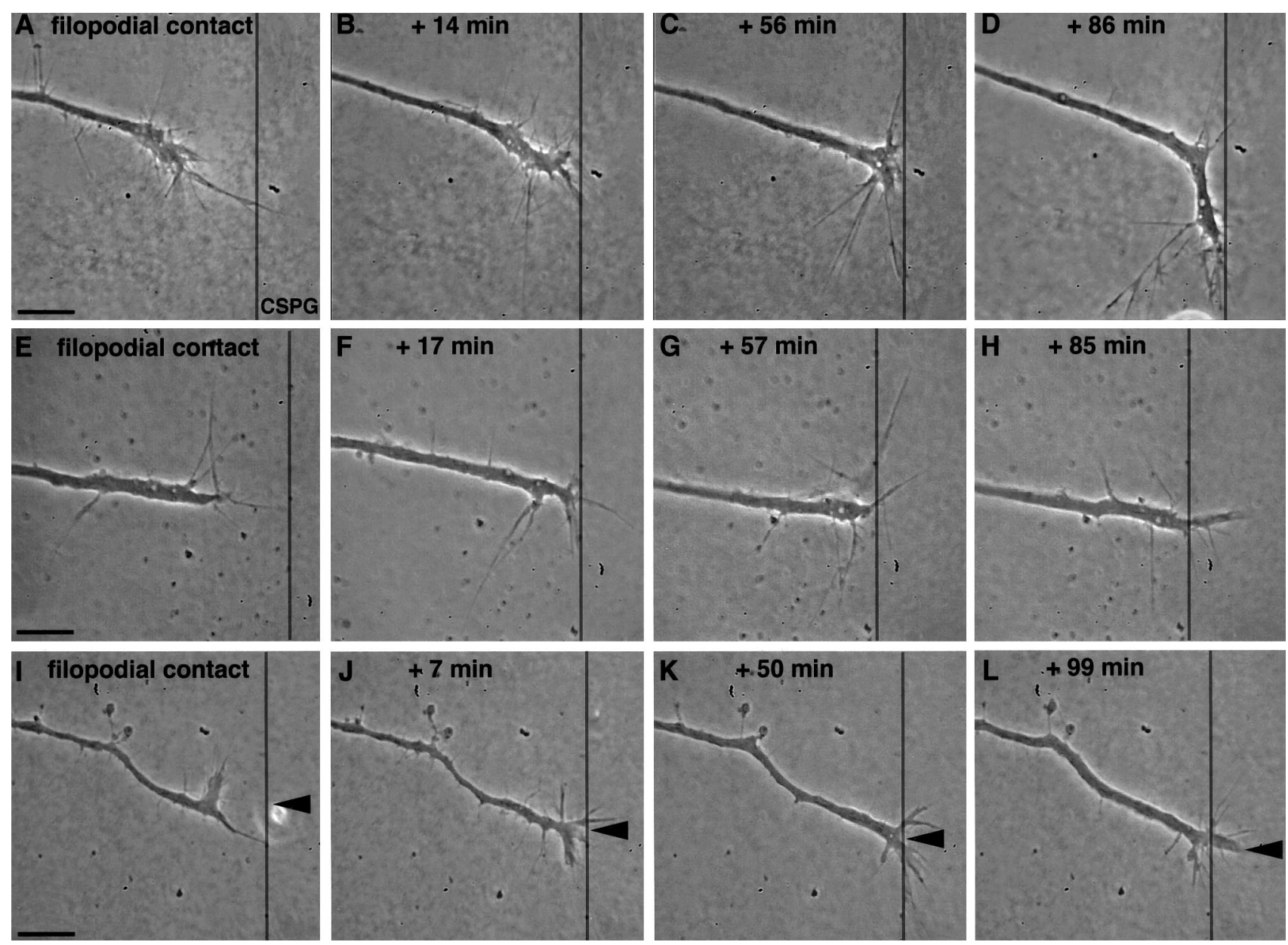

Figure 1. Phase-contrast video sequences showing a control growth cone that turned to avoid a CSPG border compared with vinblastine- and taxol-treated growth cones that failed to turn. $A-D$, Turning control growth cone. $E-H$, Growth cone that stopped at the border in the presence of 4 nM vinblastine. $I-L, 7 \mathrm{~nm}$ taxol-treated growth cone that sidestepped and stopped at the CSPG border. Arrowheads in $I-L$ mark the position of the taxol-treated growth cone at the border and show that it sidestepped laterally along the border for a short distance. All three sequences include the first filopodial contact with CSPG $(A, E, I)$ followed by $\mathrm{C}$-domain advance to the FN/CSPG border $(B, F, J)$, continued filopodial sampling of the CSPG stripe $(C, G, K)$, and either turning (control; $D)$ or continued interaction with the border (drug-treated; $H, L)$. Drug-treated growth cones did not turn, even after interacting with the CSPG border for periods of $4-6 \mathrm{hr}$ or overnight. For each sequence, the time interval after first filopodial contact is shown in the subsequent frames. Scale bar, $10 \mu \mathrm{m}$.

differences were assessed by either the Kruskal-Wallis test with post hoc multiple comparison (Mosteller and Rourke, 1973; Conover, 1980) or the Mann-Whitney test (Mosteller and Rourke, 1973).

\section{RESULTS}

\section{Effects of taxol and vinblastine on growth cone migration rate}

Low concentrations of taxol and vinblastine have recently been shown to be effective in reducing MT dynamics without dramatically affecting growth cone motility ( $\mathrm{Yu}$ and Baas, 1995). In preliminary experiments, we determined that in the presence of $7 \mathrm{~nm}$ taxol or $4 \mathrm{~nm}$ vinblastine, DRG growth cones continued to migrate, whereas 5- to 10-fold higher concentrations of these drugs either stopped growth cone migration or caused neurite retraction. To ascertain whether $4 \mathrm{~nm}$ vinblastine and $7 \mathrm{~nm}$ taxol had substantial effects on DRG growth cone migration and neurite elongation, we examined time-lapse video recordings and calculated the migration rates of a sample of growth cones over a 15-60 min period before addition of the drug, during drug treatment, and after washout of the drug. In a separate series of experiments, we determined the rate of growth cone migration on homogeneous $\mathrm{FN}$ in the presence of control medium containing DMSO vehicle alone. The mean growth cone migration rates in micrometers per hour are shown in Table 1. Compared with the pre-vinblastine and DMSO control migration rates, growth cones migrated 33-35\% slower during vinblastine treatment, and growth cone migration did not fully recover during the $1-2 \mathrm{hr}$ period after vinblastine washout. For taxol-treated growth cones, the mean migration rates before taxol treatment, in the presence of $7 \mathrm{nM}$ taxol, and after washout were not significantly different.

\section{Taxol and vinblastine prevent growth cone turning}

To assess the role of MT dynamics in growth cone turning, we examined the behavior of growth cones in the presence and absence of vinblastine and taxol using our in vitro guidance assay, a substratum composed of alternating stripes of FN and CSPG (Snow et al., 1990; Challacombe et al., 1996). Growth cones that were migrating on homogeneous $\mathrm{FN}$ were recorded once per minute as they approached CSPG borders. At a distance of 50-100 $\mu \mathrm{m}$ from a border, either taxol or vinblastine was added, 

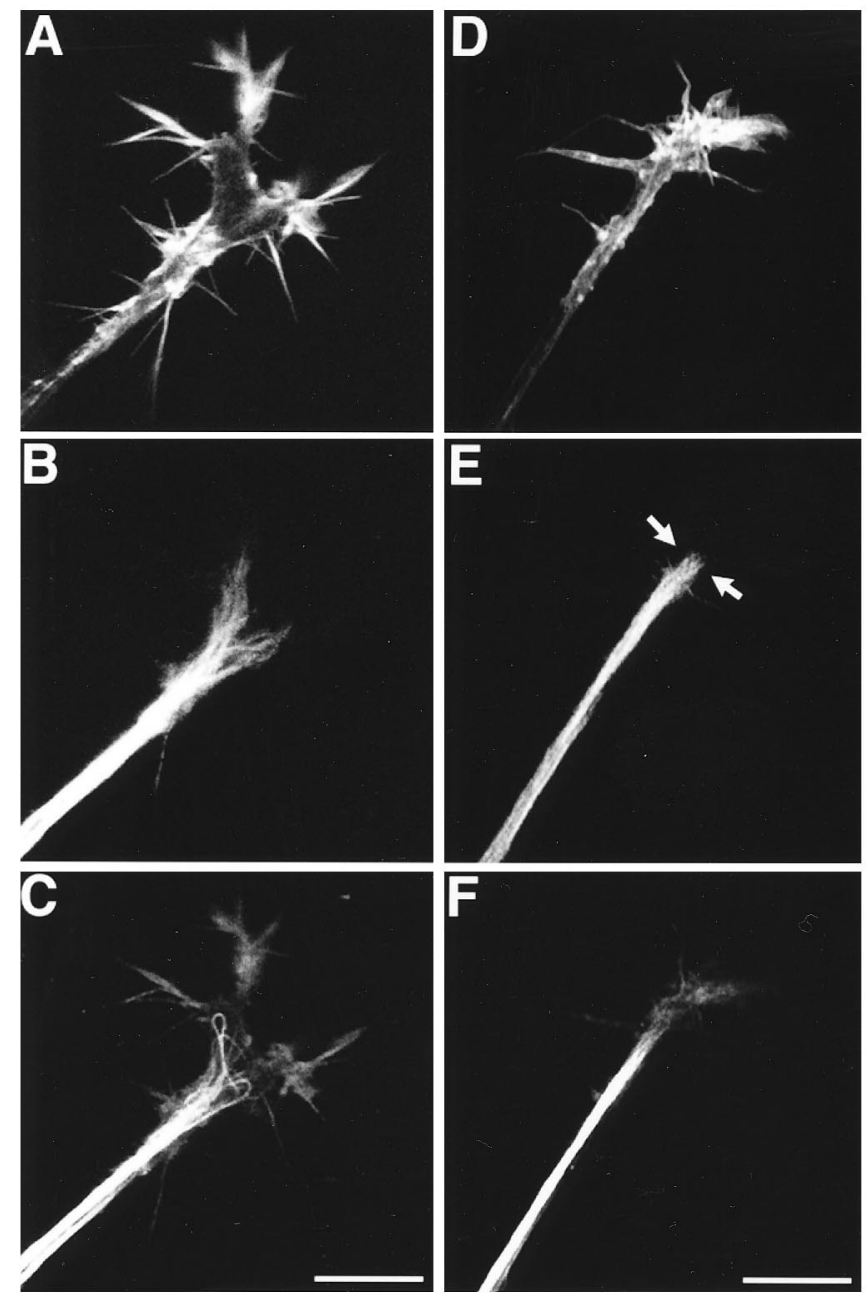
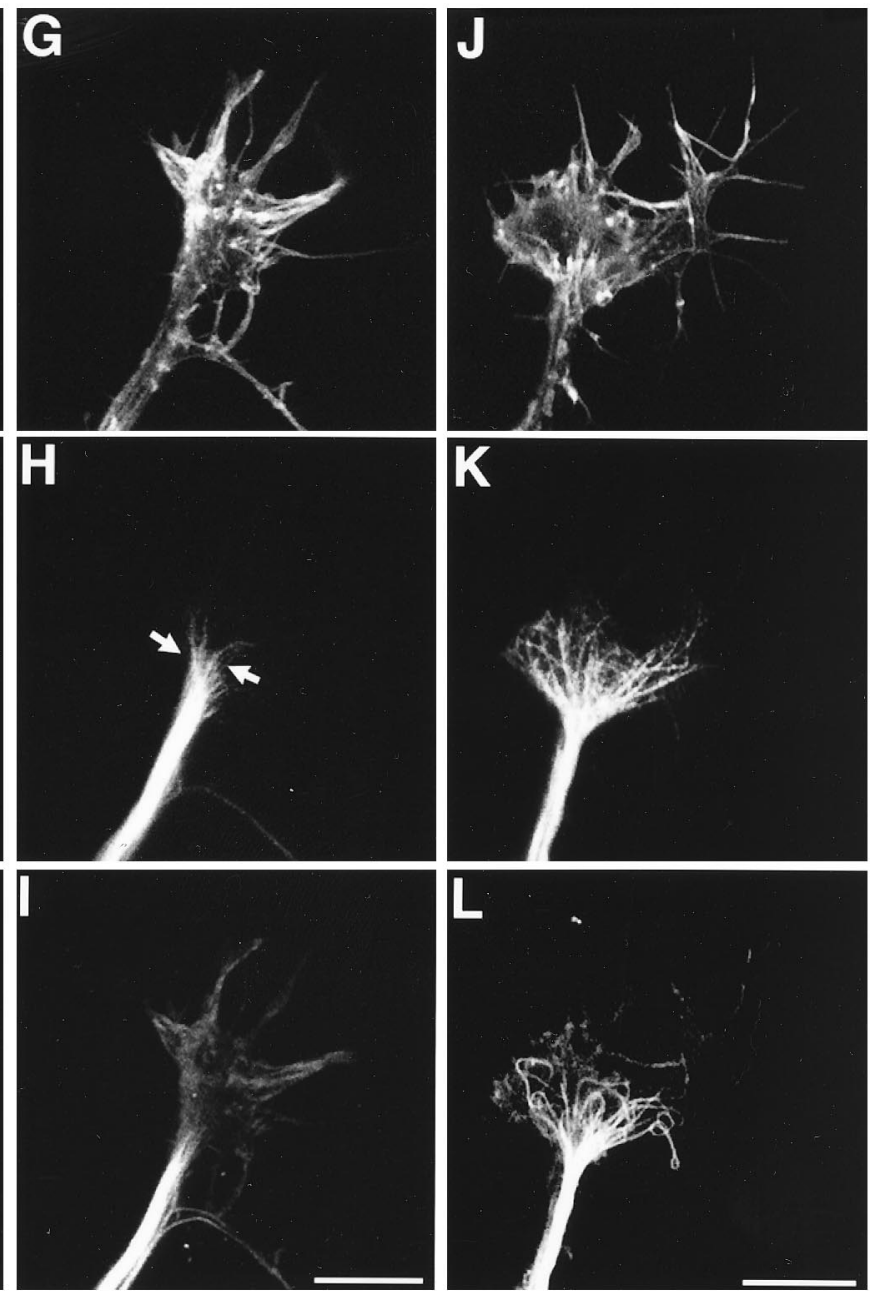

Figure 2. Comparison of AF and MT organization in chick DRG growth cones migrating on FN in the presence and absence of $4 \mathrm{nM}$ vinblastine and $7 \mathrm{~nm}$ taxol. $A, D, G, J$, Phalloidin-labeled AFs. $B, E, H, K$, Tyrosinated $\alpha$-tubulin labeling. $C, F, I, L$, Detyrosinated $\alpha$-tubulin labeling. $A-C$, Control growth cone. $D-F$, A growth cone exposed to $4 \mathrm{~nm}$ vinblastine. $G-I$, A growth cone treated with $7 \mathrm{~nm}$ taxol. $J-L$, A growth cone exposed to $4 \mathrm{~nm}$ vinblastine, which was subsequently washed out. Arrows in $E$ and $H$ point to the bundled MTs that stained with antibodies directed against tyrosinated $\alpha$-tubulin, showing that the Tyr-MT ends in the vinblastine- and taxol-treated growth cones are less splayed than those in the control growth cone. Scale bar, $10 \mu \mathrm{m}$.

and the growth cones were recorded as they advanced and then contacted CSPG, and for an additional period of up to $4 \mathrm{hr}$ after first contact. This time period was chosen because $83 \%$ of control growth cones that turn will do so within approximately $2 \mathrm{hr}$ of initial contact with the CSPG border (Challacombe et al., 1996). The behaviors of vinblastine- and taxol-treated growth cones at FN/CSPG borders are shown in Table 2 . Whereas $61 \%$ of control growth cones turned to avoid CSPG and migrated along the border (Challacombe et al., 1996), growth cones treated with the drugs did not turn. Drug-treated growth cones at CSPG borders were recorded for periods of $4-6 \mathrm{hr}$ or overnight. Many vinblastine- and taxol-treated growth cones underwent alternating periods of stopping, brief retractions, and lateral movement (sidestepping) along the CSPG border. During these behaviors, growth cone filopodia sampled the CSPG by protruding, waving, bending, and retracting in a manner similar to that of control growth cones (Snow et al., 1991, 1994; Challacombe et al., 1996). Figure 1 shows phase-contrast sequences of growth cones exhibiting normal turning behavior $(A-D)$, stopping in the presence of vinblastine $(E-$ $H)$, and stopping/sidestepping in the presence of taxol $(I-L)$. In a separate series of experiments, we determined whether vinblastine- and taxol-treated growth cones that had been stopped or sidestepping at CSPG borders for at least $1 \mathrm{hr}$ would return to normal behavior after drug washout. Results indicate that $75 \%$ of growth cones exposed to vinblastine and $57 \%$ of growth cones exposed to taxol turned within 1-2 hr after drug washout (data not shown). Taken together, these results show that low concentrations of vinblastine and taxol, which reduce MT dynamics, completely prevent growth cone turning. Furthermore, the effects of these drugs on growth cone turning are reversible.

\section{Taxol and vinblastine alter MT organization}

Because low concentrations of vinblastine and taxol prevented growth cone turning, we examined the organization of MTs and AFs in recorded growth cones that were migrating on homogeneous FN or at a CSPG border. To obtain fluorescent images of the cytoskeleton in the previously recorded growth cones, culture dishes were fixed and immunocytochemically labeled with fluorescent phalloidin and with antibodies directed against tyrosinated and detyrosinated $\alpha$-tubulin, and confocal images were acquired as described previously (Challacombe et al., 1996). De- 

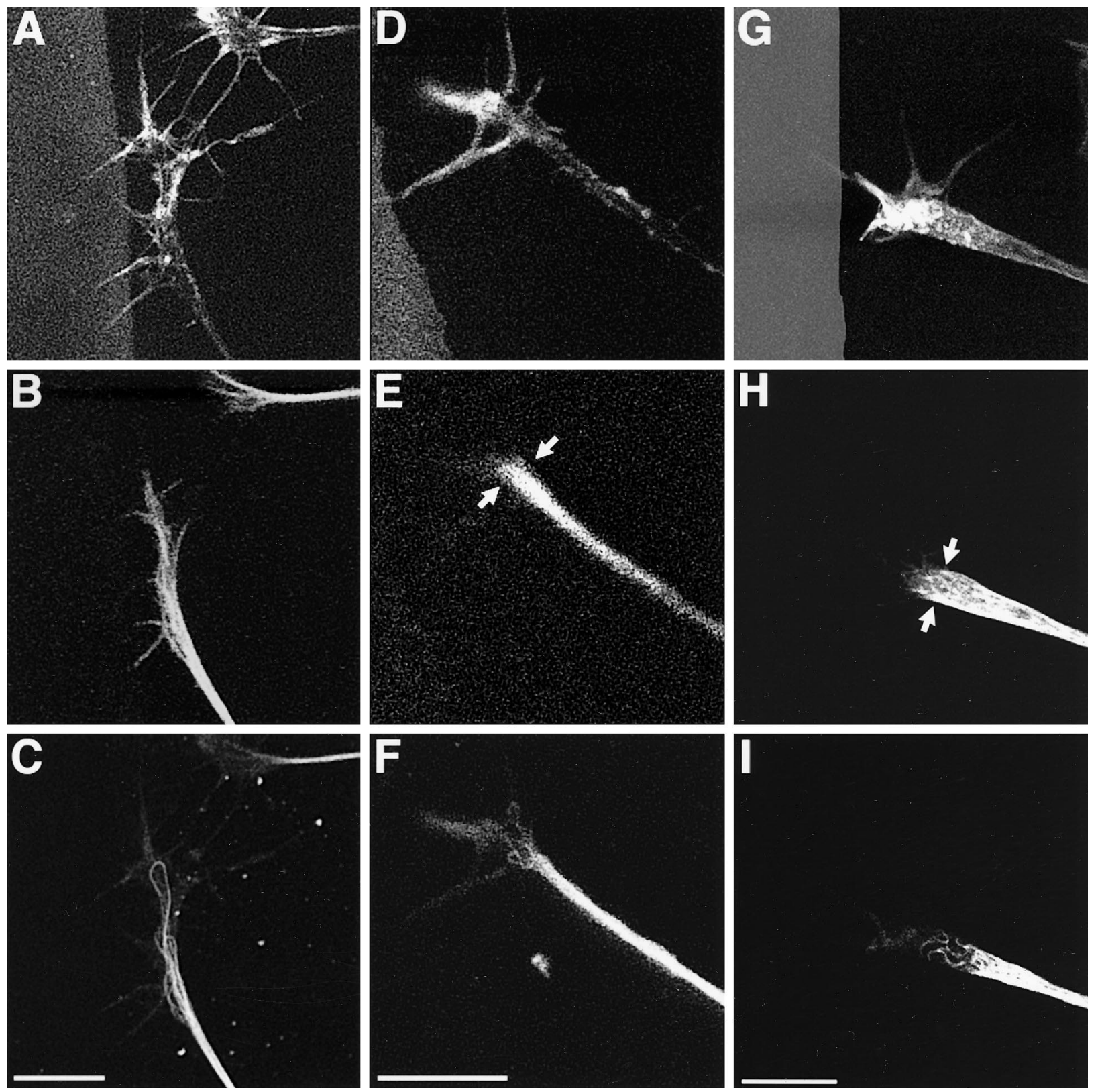

Figure 3. Comparison of AF and MT organization in chick DRG growth cones at a CSPG border in the presence and absence of 4 nM vinblastine and $7 \mathrm{~nm}$ taxol. $A, D, G$, Phalloidin-labeled AFs. $B, E, H$, Tyrosinated $\alpha$-tubulin labeling. $C, F, I$, Detyrosinated $\alpha$-tubulin labeling. $A-C$, A control growth cone turning to avoid growing on CSPG. $D-F$, A growth cone exposed to 4 nM vinblastine that has contacted the CSPG border. $G-I$, A growth cone treated with $7 \mathrm{~nm}$ taxol that is stopped at the border. The CSPG border is shown in $A, D$, and $G$. In $G$, the border was digitally enhanced to make it more visible. Vinblastine- and taxol-treated growth cones do not turn to avoid CSPG, but instead stop, retract, or sidestep laterally along the border. Arrows in $E$ and $H$ show that, in drug-treated growth cones at CSPG borders, the organization of Tyr-MTs is similar to that of drug-treated growth cones migrating on FN (see Fig. 2). Scale bar, $10 \mu \mathrm{m}$.

tyrosination is a post-translational modification of $\alpha$-tubulin, carried out by a tubulin-specific carboxypeptidase that acts on the tubulin subunits within MTs. The reverse reaction, tyrosination, is performed by tubulin tyrosine ligase, which acts on soluble tubulin subunits (for review, see Bulinski and Gundersen, 1991). As MT polymers persist in cells, they stain more strongly with antibodies against detyrosinated $\alpha$-tubulin and become less reactive with antibodies against tyrosinated $\alpha$-tubulin; thus, antibodies directed against these two different tubulin isotypes can be used to distinguish between older and newer MTs (Gundersen et al., 1984; Webster et al., 1987; Baas and Black, 1990; Arregui et al., 1991;
Baas et al., 1993; Brown et al., 1993). In this report, we refer to MTs stained with antityrosinated $\alpha$-tubulin antibodies as TyrMTs, and those labeled with antibodies directed against detyrosinated $\alpha$-tubulin as Detyr-MTs.

When untreated DRG growth cones migrate on homogeneous FN, the MT bundle extends throughout the $\mathrm{C}$-domain, and individual Tyr-MTs splay into the P-domain where they overlap with AF bundles at the bases of filopodia. In contrast to controls, in vinblastine- and taxol-treated growth cones migrating on homogeneous FN, Tyr-MTs were less splayed throughout the growth cone (Fig. 2). In control growth cones migrating 

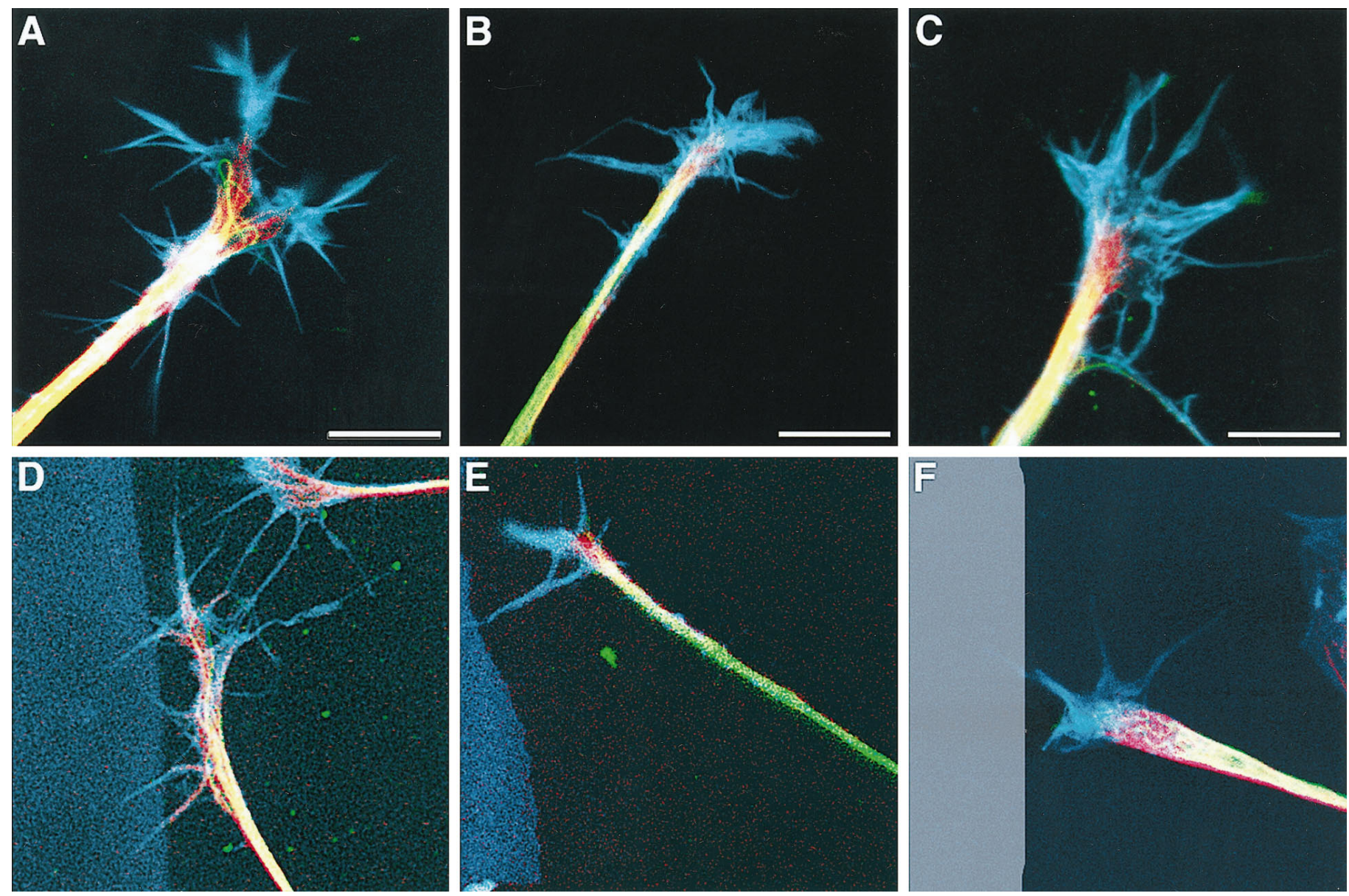

Figure 4. Merged three-color images showing the organization of AFs and MTs in growth cones migrating on FN and at CSPG borders in the presence and absence of vinblastine and taxol. $A-C$ correspond to Figure 2, and $D-F$ correspond to Figure 3 . In $F$, the CSPG border was digitally enhanced to make it more visible. In all panels, AF labeling is blue, tyrosinated $\alpha$-tubulin labeling is red, and detyrosinated $\alpha$-tubulin labeling is green. The close proximity of Tyr-MTs (red) to AFs (blue) is apparent in all growth cones, as is the reduced splaying of Tyr-MT ends in growth cones exposed to vinblastine and taxol. These color images also demonstrate that the organization of MTs in drug-treated growth cones at CSPG borders is similar to those migrating on FN. Scale bar, $10 \mu \mathrm{m}$.

on FN, phalloidin-labeled AFs were present at the leading edge and extended as bundles within filopodia (Fig. $2 A$ ), whereas the ends of Tyr-MTs (Fig. $2 B$ ) projected into the P-domain, overlapping with AFs at the bases of filopodia. Detyr-MTs were confined to the C-domain where some of them formed loops (Fig. $2 C$ ). In growth cones treated with $4 \mathrm{~nm}$ vinblastine (Fig. $2 D$ ) and $7 \mathrm{~nm}$ taxol (Fig. 2G), phalloidin-labeled AFs were present at the leading edge and in filopodia. However, Tyr-MTs (Fig. 2E,H, arrows) were less splayed than in control growth cones as they extended forward into the AF-rich P-domain. Another difference between control and drug-treated growth cones was that in control growth cones, Detyr-MTs formed loops in the C-domain (Fig. 2C), whereas in vinblastine- (Fig. $2 F$ ) and taxol-treated (Fig. 2I) growth cones, such looped Detyr-MTs were less prevalent. In growth cones migrating on FN and exposed to vinblastine or taxol for 1-2 hr followed by washout of the drugs for 1-2 hr (Fig. $2 J-L)$, the MTs were even more splayed than in controls. In a representative growth cone exposed to vinblastine, which was subsequently washed out, phalloidin-labeled AFs were present at the leading edge (Fig. $2 J)$, the ends of Tyr-MTs were widely splayed throughout the P-domain (Fig. 2K), and Detyr-MTs formed loops in the C-domain (Fig. 2L).
Figure 3 compares the organization of AFs and MTs in a control growth cone turning at a CSPG border with vinblastineand taxol-treated growth cones that were either stopped or sidestepping at a CSPG border. In the turning control growth cone (Fig. $3 A-C$ ), the ends of Tyr-MTs (Fig. $3 B$ ) and Detyr-MTs (Fig. $3 C$ ) are bundled and turned to be aligned along the border, with Tyr-MT ends projecting into the bases of AF-rich filopodia at the growth cone tip (see also Fig. 4D). The arrangement of MTs and AFs in vinblastine- and taxol-treated growth cones at a CSPG border is shown in Figure 3D-I. The organization of Tyr-MTs (Fig. 3E,H) and Detyr-MTs (Fig. $3 F, I$ ) within the growth cones is similar to the MT organization in the drug-treated growth cones migrating on $\mathrm{FN}$ (Fig. 2E,F,H,I).

A more direct comparison of the MT distribution between vinblastine- and taxol-treated growth cones and controls can be made by examining the three-color images in Figure 4. $A-C$ correspond to Figure 2, and $D-F$ correspond to Figure 3 . The splaying and close proximity of Tyr-MT ends (red) to AFs (blue) are apparent in the control growth cones (Fig. $4 A, D$ ), as is the reduced splaying of Tyr-MT ends (red) in the vinblastine- (Fig. 4B) and taxol-treated (Fig. $4 C$ ) growth cones migrating on FN. Figure 4 also illustrates the bundling and turning of the MTs in the control growth cone that was turning at a CSPG border (Fig. 4D), whereas the configuration of 


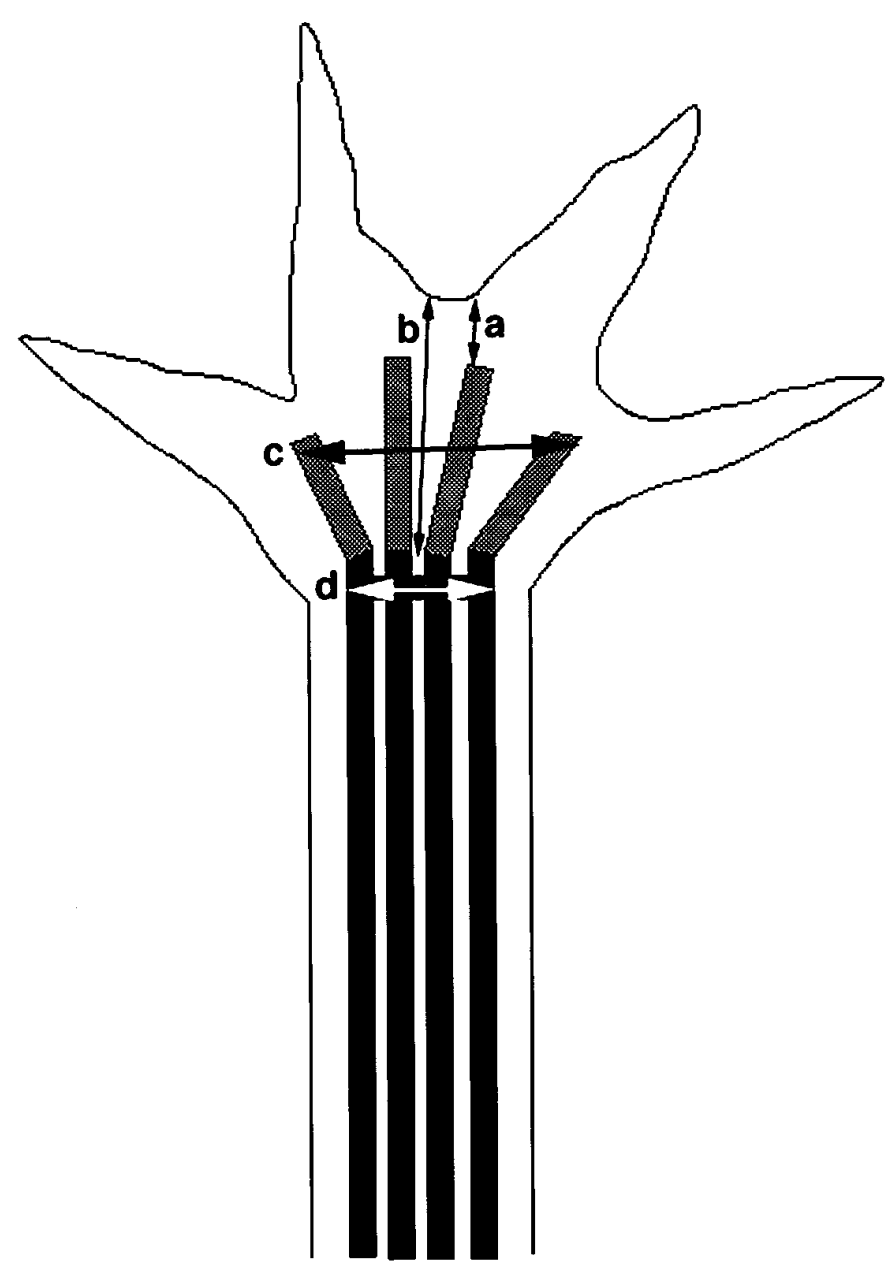

Figure 5. Schematic diagram of a growth cone illustrating how measurements of MT organization were made. Detyr-MTs are shown in black, and Tyr-MT ends are gray. Measurements from the leading edge (excluding filopodia) to the distal extents of Tyr-MTs and Detyr-MTs are depicted by the thin black double-headed arrows marked $a$ and $b$, respectively. The width of the Tyr-MT ends is represented by a thicker black double-headed arrow $(c)$, and the width of Detyr-MT staining is illustrated by a white double-headed arrow $(d)$. The width of the growth cone (not marked) was from side to side at the widest point, excluding filopodia.

Tyr-MTs in the vinblastine- (Fig. 4E) and taxol-treated (Fig. 4F) growth cones that were not turning is similar to that of drug-treated growth cones migrating on FN.

\section{Quantitative analysis of MT organization in vinblastine- and taxol-treated growth cones}

In a previous study, we quantitatively analyzed the organization of Tyr-MTs, Detyr-MTs, and AFs in turning growth cones and in growth cones that failed to turn after treatment with cytochalasin B (Challacombe et al., 1996). In this study, we used a similar technique to examine the organization of Tyr-MTs and DetyrMTs in the presence of taxol and vinblastine. We measured and compared several parameters in growth cones migrating on homogeneous FN and at CSPG borders: (1) distance from the leading edge, excluding filopodia, to distal ends of Tyr-MT staining; (2) distance from leading edge to distal extents of Detyr-MT staining; (3) width of Tyr-MT staining at the widest point; (4) width of Detyr-MT staining at the widest point; and (5) width of the growth cone at the widest point. The schematic in Figure 5 illustrates how measurements 1-4 were made. The width of the growth cone was measured across the widest point, excluding filopodia.

Results of this analysis (Table 3) showed that, for growth cones migrating on homogeneous FN, the mean widths of Tyr-MT staining in vinblastine- and taxol-treated growth cones were significantly decreased compared with control growth cones. In addition, the mean growth cone widths also decreased. These results indicate that under the influence of vinblastine and taxol, the distal Tyr-MT ends are significantly less splayed in the P-domain, and the growth cones are narrower than control growth cones. Vinblastine and taxol treatment also resulted in a significant decrease in the distance from the leading edge to the distal extents of Tyr-MTs but not Detyr-MTs, indicating that the distance between the distal extents of Tyr- and Detyr-MTs was greater than in controls. However, the distal limit of detyrosination may not indicate the limit of MT stability in the presence of these drugs, because the Tyr-MT ends may become stabilized before Tyr-tubulin is accessible to the enzyme responsible for detyrosination. A significant observation was that when taxol and vinblastine were washed out, growth cones became significantly wider than even the control growth cones, and the splayed width of Tyr-MT ends was also increased significantly beyond controls. This observation supports a link between the dynamic growth and shrinkage of the distal MT ends and a splayed MT distribution in the P-domain of growth cones.

Table 3 also shows a similar analysis of these MT parameters in growth cones at a CSPG border. Controls were turning, and vinblastine- and taxol-treated growth cones were either stopped at a border or sidestepping along it before fixation. Results of this analysis show that the altered MT organization induced by vinblastine and taxol treatment persists in growth cones interacting with a CSPG border. The distance from the leading edge to Tyr-MTs is significantly smaller than in controls, and the distance from the leading edge to Detyr-MTs is similar to controls. Furthermore, the widths of Tyr- and Detyr-MT staining, as well as the widths of drug-treated growth cones, are similar to controls. One of the key changes in growth cone morphology and MT organization that occurs during normal turning behavior is the bundling of the MT ends and narrowing of the growth cone (Tanaka and Kirschner, 1995; Challacombe et al., 1996). Our present findings show that for growth cones on homogeneous FN, the width of distal Tyr-MT staining and the growth cone width of control and drug-treated growth cones are significantly different, whereas in growth cones at a CSPG border, there are no differences in these parameters. Taken together, these results indicate that in vinblastine- and taxol-treated growth cones, Tyr-MT ends are less splayed and are closer to the leading edge while the growth cones are migrating on homogeneous FN, and the MT organization remains that way at a CSPG border, where the growth cones fail to turn.

\section{DISCUSSION}

The goal of this study was to examine the role of dynamic MTs in growth cone turning by using low concentrations of vinblastine and taxol. Vinblastine and taxol are unrelated plant metabolites that suppress MT dynamics at low substoichiometric concentrations, although they bind to different sites on MTs (Jordan et al., 1991, 1993). Experiments in vitro showed that taxol concentrations as low as $10 \mathrm{~nm}$ decreased both shrinking and growing events at MT ends (Derry et al., 1995). Experiments in vivo using HeLa cells showed that $8 \mathrm{~nm}$ taxol and $0.8 \mathrm{~nm}$ vinblastine inhibited mitosis by $50 \%$ without inducing significant depolymerization of 
Table 3. Quantitation of MT organization in growth cones migrating on FN and interacting with a CSPG border in the presence and absence of 4 nM vinblastine and 7 nM taxol

\begin{tabular}{|c|c|c|c|c|c|c|}
\hline Substratum & Treatment & $\begin{array}{l}\text { Leading edge } \\
\text { to Tyr-MTs }\end{array}$ & $\begin{array}{l}\text { Leading edge } \\
\text { to Detyr-MTs }\end{array}$ & $\begin{array}{l}\text { Width } \\
\text { Tyr-MTs }\end{array}$ & $\begin{array}{l}\text { Width } \\
\text { Detyr-MTs }\end{array}$ & $\begin{array}{l}\text { Width } \\
\text { growth cone }\end{array}$ \\
\hline \multirow[t]{5}{*}{$\begin{array}{l}\text { Homogeneous } \\
\text { FN }\end{array}$} & Control & $\begin{array}{c}5.63 \pm 0.34 \\
(178)\end{array}$ & $\begin{array}{c}9.10 \pm 0.40 \\
(150)\end{array}$ & $\begin{array}{c}7.85 \pm 0.89 \\
(45)\end{array}$ & $\begin{array}{c}4.01 \pm 0.58 \\
(44)\end{array}$ & $\begin{array}{c}16.44 \pm 1.50 \\
(43)\end{array}$ \\
\hline & $\begin{array}{l}4 \mathrm{~nm} \\
\quad \text { vinblastine }\end{array}$ & $\begin{array}{c}3.04 \pm 0.54^{*} \\
(20)\end{array}$ & $\begin{array}{c}9.3 \pm 0.78 \\
(20)\end{array}$ & $\begin{array}{c}3.85 \pm 0.54^{*} \\
(15)\end{array}$ & $\begin{array}{c}2.43 \pm 0.23 \\
(15)\end{array}$ & $\begin{array}{c}7.47 \pm 1.03^{*} \\
(15)\end{array}$ \\
\hline & $7 \mathrm{~nm}$ taxol & $\begin{array}{c}2.57 \pm 0.50^{*} \\
(16)\end{array}$ & $\begin{array}{c}8.72 \pm 0.77 \\
(16)\end{array}$ & $\begin{array}{c}2.88 \pm 0.44^{*} \\
(15)\end{array}$ & $\begin{array}{c}3.14 \pm 0.60 \\
(17)\end{array}$ & $\begin{array}{c}7.83 \pm 1.01^{*} \\
(15)\end{array}$ \\
\hline & $\begin{array}{c}\text { Vinblastine } \\
\text { washout }\end{array}$ & $\begin{array}{c}3.77 \pm 0.69 \\
(23)\end{array}$ & $\begin{array}{c}7.80 \pm 0.96 \\
(22)\end{array}$ & $\begin{array}{c}13.64 \pm 2.90^{* *} \\
(11)\end{array}$ & $\begin{array}{c}8.84 \pm 2.31^{* *} \\
(11)\end{array}$ & $\begin{array}{c}22.94 \pm 3.06^{* * *} \\
(11)\end{array}$ \\
\hline & $\begin{array}{l}\text { Taxol } \\
\text { washout }\end{array}$ & $\begin{array}{c}4.28 \pm 0.54 \\
(27)\end{array}$ & $\begin{array}{c}9.37 \pm 0.67 \\
(25)\end{array}$ & $\begin{array}{c}13.73 \pm 2.38^{* *} \\
(10)\end{array}$ & $\begin{array}{c}7.37 \pm 1.24^{* *} \\
(10)\end{array}$ & $\begin{array}{c}26.86 \pm 2.51^{* *} \\
(10)\end{array}$ \\
\hline \multirow[t]{3}{*}{ CSPG border } & $\begin{array}{l}\text { Control } \\
\text { turning }\end{array}$ & $\begin{array}{c}4.66 \pm 0.38 \\
(107)\end{array}$ & $\begin{array}{c}7.94 \pm 0.49 \\
(92)\end{array}$ & $\begin{array}{c}4.39 \pm 0.46 \\
(41)\end{array}$ & $\begin{array}{c}2.17 \pm 0.20 \\
(41)\end{array}$ & $\begin{array}{c}9.25 \pm 0.97 \\
(41)\end{array}$ \\
\hline & $\begin{array}{l}4 \mathrm{~nm} \\
\text { vinblastine }\end{array}$ & $\begin{array}{c}1.76 \pm 0.50^{*} \\
(20)\end{array}$ & $\begin{array}{c}6.80 \pm 0.65 \\
(20)\end{array}$ & $\begin{array}{c}3.80 \pm 0.59 \\
(21)\end{array}$ & $\begin{array}{c}2.46 \pm 0.29 \\
(21)\end{array}$ & $\begin{array}{c}8.27 \pm 1.10 \\
(19)\end{array}$ \\
\hline & $7 \mathrm{~nm}$ taxol & $\begin{array}{c}1.72 \pm 0.65^{*} \\
(20)\end{array}$ & $\begin{array}{c}6.22 \pm 0.75 \\
(20)\end{array}$ & $\begin{array}{c}5.01 \pm 1.59 \\
(15)\end{array}$ & $\begin{array}{c}3.61 \pm 1.17 \\
(15)\end{array}$ & $\begin{array}{c}7.63 \pm 0.91 \\
(13)\end{array}$ \\
\hline
\end{tabular}

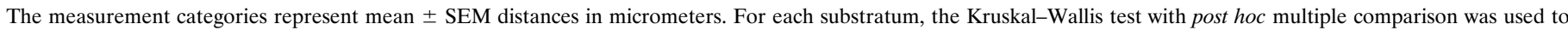

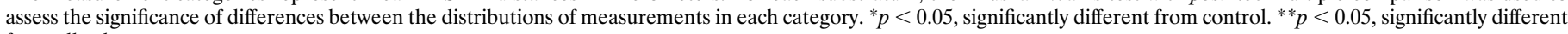
from all other groups.

MTs (Jordan et al., 1991, 1993). These results indicate that very low concentrations of taxol and vinblastine are effective in suppressing MT dynamics in cells. Thus, we used taxol and vinblastine to reduce the dynamics of MT ends in DRG growth cones and investigate the role of dynamic MTs in growth cone turning.

When control growth cones migrate to and contact a CSPG border, they turn or stop to avoid crossing the border (Challacombe et al., 1996). When we applied $7 \mathrm{nM}$ taxol or $4 \mathrm{~nm}$ vinblastine while growth cones were approaching a CSPG border, the growth cones continued to migrate toward the border, although the rate of migration was reduced by $4 \mathrm{~nm}$ vinblastine. Once at the border, none of the drug-treated growth cones turned. Instead, growth cones either stopped at the border, sidestepped laterally along the border, or exhibited a combination of behaviors that included intermittent pausing, retraction, and sidestepping without turning. Filopodia and lamellipodia continued to be actively protruded along and across the border by the drug-treated growth cones, but the growth cones never turned at the border.

Previous studies used low concentrations of MT-specific drugs to investigate the role of dynamic MTs in neurite elongation. Low doses of vinblastine and nocodazole inhibited axonal elongation from Xenopus neural tube (Tanaka et al., 1995) and rat superior cervical ganglion neurons (Rochlin et al., 1996). In contrast, we found that chick DRG neurites elongated in the presence of low concentrations of vinblastine and taxol, and a similar study showed that chick DRG neurites elongated when net MT assembly was inhibited by $4 \mathrm{~nm}$ vinblastine (Yu and Baas, 1995). These results indicate that MT translocation alone can support the elongation of DRG neurites. This conflicts with previous studies of fluorescence recovery after photobleaching of fluorescent MTs (Lim et al., 1990; Edson et al., 1993), which found that MT translocation is not significant along chick DRG neurites. However, these previous studies also found that fluorescence recovery was rapid in the distal neurite near the growth cone (Edson et al., 1993), and perhaps localized MT translocation at the distal neurite can support growth cone migration and neurite elongation for some time in the presence of anti-MT drugs.

Our results do show that dynamic MTs in the growth cone are needed for responses to guidance cues, such as turning away from CSPG. In support of this idea, observations of MT ends in living cells indicate that MT polymerization is responsible, at least in part, for advancing MTs from the growth cone C-domain into the P-domain (Tanaka et al., 1995; Miller and Joshi, 1996). A model for growth cone steering suggests that these MT ends in the P-domain are the pioneers of axonal growth that lead the advance of other MTs and neuritic components (Sabry et al., 1991; Tanaka and Kirschner, 1991; Lin and Forscher, 1993; Bentley and O'Connor, 1994; Tanaka and Kirschner, 1995). Recent evidence indicates that dynamic MTs play a role in growth cone turning at a laminin/tenascin border in vitro (Williamson et al., 1996). We propose that a key event in growth cone turning or branching may be the capture or stabilization of dynamic MT ends at one side of the growth cone via their interactions with AF bundles of lamellipodia and filopodia (Letourneau and Ressler, 1983; Forscher and Smith, 1988; Gordon-Weeks, 1991; Tanaka and Kirschner, 1991; Lin and Forscher, 1993; Challacombe et al., 1996). In a previous study, we tested this hypothesis with the AF-depolymerizing drug cytochalasin $\mathrm{B}$ and found that, in the absence of AF bundles, MT ends in the P-domain were widely splayed. When such growth cones reached a border with a repulsive substratum, the MTs were not steered to one side to initiate growth cone turning (Challacombe et al., 1996). Our present results provide substantial evidence in support of the idea that dynamic MT ends are required for growth cone turning to avoid an inhibitory guidance cue. Our observations and measurements revealed two major differences in the distribution of MTs between taxol- and vinblastine-treated growth cones and 


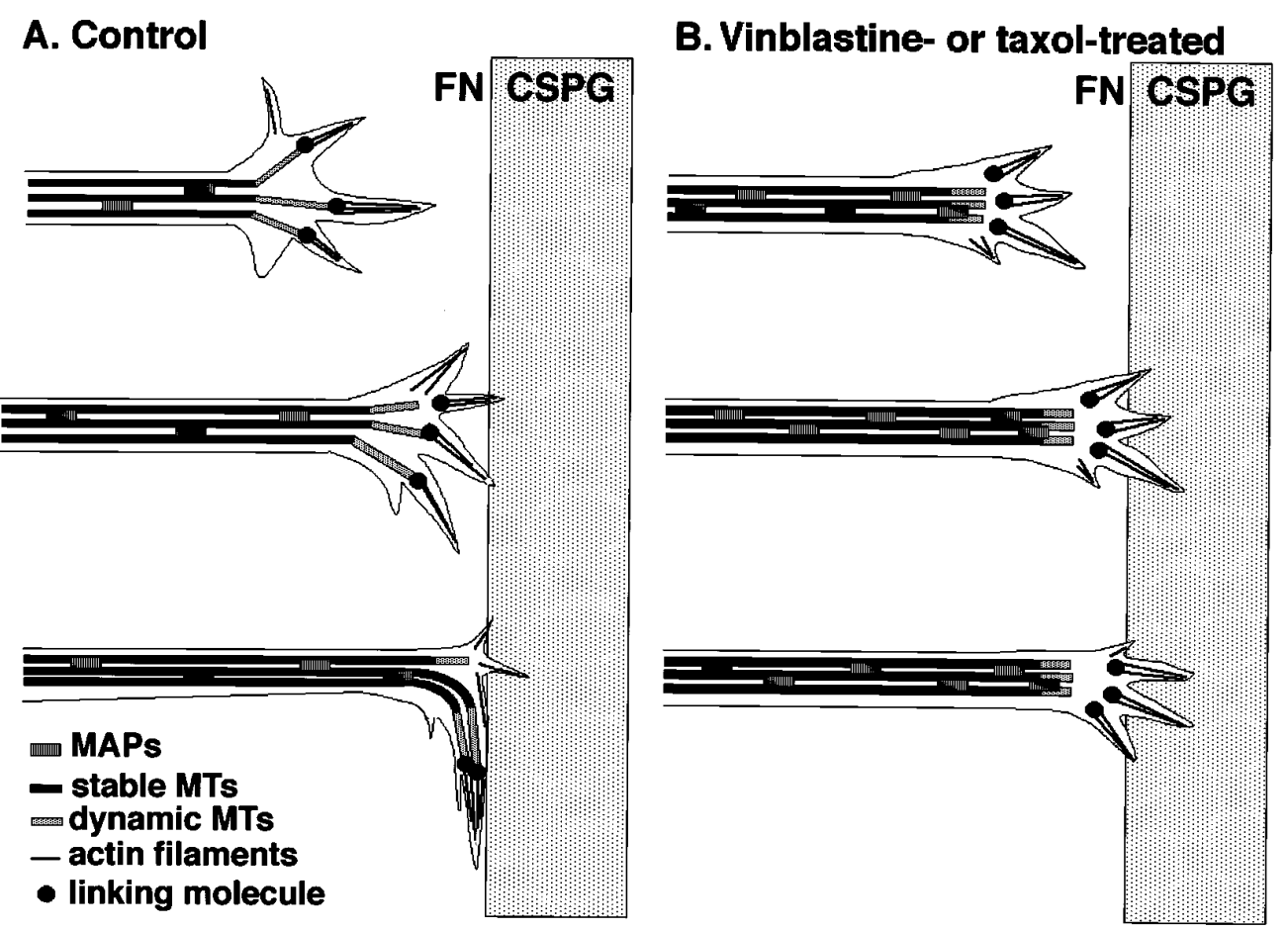

Figure 6. A, A model for growth cone turning. In a growth cone migrating on homogeneous FN, stable MTs (black) are bundled in the neurite by the activity of MAPs (striped rectangles) and extend into the C-domain, whereas dynamic MT ends (gray) splay throughout the P-domain. Some dynamic MT ends are linked to AF bundles (thin black lines) at the bases of filopodia by an unknown linkage molecule ( filled circles). When the growth cone contacts a CSPG border, filopodia sample the CSPG, but these are unstable contacts. Some filopodia remain on FN, forming more stable contacts with the substratum. When the AF bundles of these filopodia are linked to dynamic MT ends, the AF bundles steer the dynamic MT ends asymmetrically along the border to initiate the turn. During turning, the growth cone MTs become bundled and aligned along the border. $B, A$ growth cone that fails to turn in the presence of low concentrations of taxol or vinblastine. In a growth cone treated with taxol or vinblastine, the MT ends in the $\mathrm{P}$-domain are less dynamic and become less splayed while the growth cone is migrating on FN. When the growth cone contacts a CSPG border, filopodia sample the CSPG but the distal MTs remain less splayed in the P-domain, the MT ends are not steered by AFs, and the growth cone fails to turn. This may be attributable to a premature stabilization and stiffening of the MT ends in the P-domain, resulting either directly from drug-induced changes in MT properties or from increased binding of MAPs to the less dynamic MTs. The failure to turn also may be because of an inability of the linkage molecule to connect AFs to the less dynamic MT ends. control growth cones on homogeneous FN. The ends of TyrMTs were closer to the leading growth cone margin and were less splayed than in control growth cones. In addition, drugtreated growth cones were narrower than controls.

Our data indicate that vinblastine- and taxol-treated growth cones fail to turn because their MT organization is altered such that MTs are not steered to one side. How does the reduction in MT dynamics by vinblastine and taxol interfere with the MT rearrangements that are crucial for growth cone turning? One possibility is that, because the MTs of vinblastine- and taxol-treated growth cones are less splayed in the P-domain, the MT ends may not interact to a sufficient degree with AF bundles, and are therefore not steered to one side to initiate a growth cone turn (Fig. 6).

The reduced dynamics of these MT ends may lead to a premature stabilization and stiffening of the MT ends in the P-domain, so they assume the bundled configuration that is seen in the C-domain and more proximal growth cone (Fig. $6 B$ ). This reduced splaying of the MT ends may result either directly from drug-induced changes in MT properties (Mickey and Howard, 1995; Feigner et al., 1996) or perhaps from facilitated binding of MAPs to the less dynamic MT ends (Black, 1987; Chapin et al., 1991; Chapin and Bulinski, 1992; Dreschel et al., 1992; Matus, 1994; Heidemann, 1996). As a result, the pull exerted by AFs cannot overcome the increased stiffness and interactions between the MT ends.

Based on results from other studies of the effects of MT drugs on cell motility, we suggest that another contribution to the inhibition of growth cone turning by these drugs is that dynamic MT ends are necessary for active remodeling of AF networks in the growth cone P-domain. Bershadsky et al. (1991) showed that MT-binding drugs reduced the protrusive activity of fibroblasts by acting on MT organization and not by a direct action on actin. Our findings that drug-treated growth cones are narrower, and that Tyr-MTs are closer to the leading edge suggest that vinblastine and taxol reduced the size of the P-domain. Similarly, our previous study also showed that the filopodia of taxol-treated growth cones extended across a narrower range of angles than control growth cone filopodia (Letourneau et al., 1986). Therefore, the effects of vinblastine and taxol on MT dynamics may have indirectly reduced the tension and ability of AFs to pull on MT ends in the P-domain.

We noted that when taxol and vinblastine were removed from the culture medium of growth cones migrating on FN, the distal Tyr-MT ends recovered and became widely splayed in the P-domain, and growth cones were wider than control growth cones. This result supports our hypothesis that the splayed configuration of MT ends in the P-domain of growth cones on FN requires dynamic MT ends (but not AF bundles, because cytochalasin B-treated growth cones contain widely splayed MTs) (Challacombe et al., 1996).

In summary, we found that low concentrations of taxol and vinblastine, which reduce MT dynamics, prevent DRG growth 
cone turning. These results support the hypothesis proposed by Tanaka et al. (1995), that dynamic MT ends play a key role in the MT rearrangements that occur during growth cone migration and axonal elongation. Furthermore, we provide important new data suggesting that dynamic MT ends are necessary for the MT rearrangements in the P-domain that underlie growth cone turning. Taken together with our previous finding that AF bundles direct MT bundling and reorientation during turning (Challacombe et al., 1996), our present results suggest that chick DRG growth cone turning to avoid CSPG depends on the presence of dynamic MT ends in the P-domain, where they splay and are captured by filopodial AFs (Fig. 6 $A$ ). The steering of these dynamic MT ends to one side leads to the asymmetric alignment of MTs as the growth cone turns along the CSPG border.

\section{REFERENCES}

Arregui C, Busciglio A, Caceres A, Barra HS (1991) Tyrosinated and detyrosinated microtubules in axonal processes of cerebellar macroneurons grown in culture. J Neurosci Res 28:171-181.

Avila J, Dominguez J, Diaz-Nido J (1994) Regulation of microtubule dynamics by microtubule-associated protein expression and phosphorylation during neuronal development. Int J Dev Biol 38:13-25.

Baas PW, Black MM (1990) Individual microtubules in the axon consist of domains that differ in both composition and stability. J Cell Biol 111:495-509.

Baas PW, Ahmad FJ, Pienkowski TP, Brown A, Black MM (1993) Sites of microtubule stabilization for the axon. J Neurosci 13:2177-2185.

Bentley D, O'Connor TP (1994) Cytoskeletal events in growth cone steering. Curr Opin Neurobiol 4:43-48.

Bershadsky AD, Vaisberg EA, Vasiliev JM (1991) Pseudopodial activity at the active edge of migrating fibroblasts is decreased after drug-induced microtubule depolymerization. Cell Motil Cytoskeleton 19:152-158.

Black MM (1987) Taxol interferes with the interaction of microtubuleassociated proteins with microtubules in cultured neurons. J Neurosci 7:3695-3702.

Brelje TC, Wessendorf MW, Sorenson RL (1993) Multicolor laser scanning confocal immunofluorescence microscopy: practical applications and limitations. Methods Cell Biol 38:97-181.

Brown A, Li Y, Slaughter T, Black MM (1993) Composite microtubules of the axon: quantitative analysis of tyrosinated and acetylated tubulin along individual axons. J Cell Sci 104:339-352.

Bulinski JC, Gundersen GG (1991) Stabilization and post-translational modification of microtubules during cellular morphogenesis. BioEssays 13:285-292.

Carrino DA, Kaplan AI (1985) Isolation and characterization of proteoglycans synthesized in ovo by embryonic chick cartilage and new bone. J Biol Chem 260:122-127.

Challacombe JF, Snow DM, Letourneau PC (1996) Actin filament bundles are required for microtubule reorientation during growth cone turning to avoid an inhibitory guidance cue. J Cell Sci 109:2031-2040.

Chapin SJ, Bulinski JC (1992) Microtubule stabilization by assemblypromoting microtubule-associated proteins: a repeat performance. Cell Motil Cytoskeleton 23:236-243.

Chapin SJ, Bulinski JC, Gundersen GG (1991) Microtubule bundling in cells. Nature 349:24.

Conover WJ (1980) Practical nonparametric statistics, 2nd Ed. New York: Wiley.

Davis A, Sage CR, Dougherty CA, Farrell KW (1994) Microtubule dynamics modulated by guanosine triphosphate hydrolysis activity of $\beta$-tubulin. Science 264:839-841.

Derry WB, Wilson L, Jordan MA (1995) Substoichiometric binding of taxol suppresses microtubule dynamics. Biochemistry 34:2203-2211.

Dreschel DN, Hyman AA, Cobb MH, Kirschner MW (1992) Modulation of the dynamic instability of tubulin by the microtubule-associated protein tau. Mol Biol Cell 3:1141-1154.

Edson KJ, Lim S-S, Borisy GG, Letourneau PC (1993) FRAP analysis of the stability of the microtubule population along the neurites of chick sensory neurons. Cell Motil Cytoskeleton 25:59-72.
Feigner H, Frank R, Schliwa M (1996) Flexural rigidity of microtubules measured with the use of optical tweezers. J Cell Sci 109:509-516.

Forscher P, Smith SJ (1988) Actions of cytochalasins on the organization of actin filaments and microtubules in a neuronal growth cone. J Cell Biol 107:1505-1516.

Gordon-Weeks PR (1991) Evidence for microtubule capture by filopodial actin filaments in growth cones. NeuroReport 2:573-576.

Gundersen GG, Kalnoski MH, Bulinski JC (1984) Distinct populations of microtubules: tyrosinated and nontyrosinated alpha tubulin are distributed differently in vivo. Cell 38:779-789.

Heidemann SR (1996) Cytoplasmic mechanisms of axonal and dendritic growth in neurons. Int Rev Cytol 165:235-296.

Jordan MA, Thrower D, Wilson L (1991) Mechanism of inhibition of cell proliferation by vinca alkaloids. Cancer Res 51:2212-2222.

Jordan MA, Thrower D, Wilson L (1992) Effects of vinblastine, podophyllotoxin and nocodazole on mitotic spindles. Implications for the role of microtubule dynamics in mitosis. J Cell Sci 102:401-416.

Jordan MA, Toso RJ, Thrower D, Wilson L (1993) Mechanism of mitotic block and inhibition of cell proliferation by taxol at low concentrations. Proc Natl Acad Sci USA 90:9552-9556.

Kilmartin JV, Wright B, Milstein D (1982) Rat monoclonal antitubulin antibodies derived using a new non-secretory rat cell line. J Cell Biol 9:3576-3582.

Lagenaur C, Lemmon V (1987) An L1-like molecule, the 8D9 antigen, is a potent substrate for neurite extension. Proc Natl Acad Sci USA 84:7753-7757.

Letourneau PC, Ressler AH (1983) Differences in the organization of actin in the growth cones compared with the neurites of cultured neurons from chick embryos. J Cell Biol 97:963-973.

Letourneau PC, Ressler AH (1984) Inhibition of neurite initiation and growth by taxol. J Cell Biol 98:1355-1362.

Letourneau PC, Shattuck TA, Ressler AH (1986) Branching of sensory and sympathetic neurites in vitro is inhibited by treatment with taxol. J Neurosci 6:1912-1917.

Letourneau PC, Shattuck TA, Roche FK, Takeichi M, Lemmon V (1990) Nerve growth cone migration onto Schwann cells involves the calcium-dependent adhesion molecule, N-cadherin. Dev Biol 138:430-442.

Lewis AK, Bridgman PC (1992) Nerve growth cone lamellipodia contain two populations of actin filaments that differ in organization and polarity. J Cell Biol 119:1219-1243.

Liao G, Nagasaki T, Gundersen GG (1995) Low concentrations of nocodazole interfere with fibroblast locomotion without significantly affecting microtubule level: implications for the role of dynamic microtubules in cell locomotion. J Cell Sci 108:3473-3483.

Lim S-S, Edson KJ, Letourneau PC, Borisy GG (1990) A test of microtubule translocation during neurite elongation. J Cell Biol 111:123-130.

Lin C-H, Forscher P (1993) Cytoskeletal remodeling during growth cone-target interactions. J Cell Biol 121:1369-1383.

Matus A (1994) Stiff microtubules and neuronal morphology. Trends Neurosci 17:19-22.

Mickey B, Howard J (1995) Rigidity of microtubules is increased by stabilizing agents. J Cell Biol 130:909-917.

Miller KE, Joshi HC (1996) Tubulin transport in neurons. J Cell Biol 133:1355-1366.

Mitchison T, Kirschner M (1984) Dynamic instability of microtubule growth. Nature 312:237-242.

Mosteller F, Rourke REK (1973) Sturdy statistics. Nonparametrics and order statistics. Reading, MA: Addison-Wesley.

Panda D, Miller HP, Banerjee A, Luduena RF, Wilson L (1994) Microtubule dynamics in vitro are regulated by the tubulin isotype composition. Proc Natl Acad Sci USA 91:11358-11362.

Rochlin MW, Wickline KM, Bridgman PC (1996) Microtubule stability decreases axon elongation but not axoplasm production. J Neurosci 16:3236-3246.

Sabry JH, O'Connor TP, Evans L, Toroian-Raymond A, Kirschner M, Bentley D (1991) Microtubule behavior during guidance of pioneer neuron growth cones in situ. J Cell Biol 115:381-395.

Schliwa M, van Blerkom J (1981) Structural interaction of cytoskeletal components. J Cell Biol 90:222-235.

Snow DM, Lemmon V, Carrino DA, Caplan AI, Silver J (1990) Sulfated proteoglycans in astroglial barriers inhibit neurite outgrowth in vitro. Exp Neurol 109:111-130.

Snow DM, Watanabe M, Letourneau PC, Silver J (1991) A chondroitin 
sulfate proteoglycan may influence the direction of retinal ganglion cell outgrowth. Development 113:1473-1485.

Snow DM, Atkinson PB, Hassinger TD, Letourneau PC, Kater SB (1994) Chondroitin sulfate proteoglycan elevates cytoplasmic calcium in DRG neurons. Dev Biol 166:87-100.

Tanaka EM, Kirschner MW (1991) Microtubule behavior in the growth cones of living neurons during axon elongation. J Cell Biol 115:345-363.

Tanaka EM, Kirschner MW (1995) The role of microtubules in growth cone turning at substrate boundaries. J Cell Biol 128:127-137.

Tanaka EM, Ho T, Kirschner MW (1995) The role of microtubule dynamics in growth cone motility and axonal growth. J Cell Biol $128: 139-155$.
Webster DR, Gundersen GG, Bulinski JC, Borisy GG (1987) Differential turnover of tyrosinated and detyrosinated microtubules. Proc Natl Acad Sci USA 84:9040-9044.

Williamson T, Gordon-Weeks PR, Schachner M, Taylor J (1996) Microtubule reorganization is obligatory for growth cone turning. Proc Natl Acad Sci USA 93:15221-15226.

Yu W, Baas PW (1995) The growth of the axon is not dependent upon net microtubule assembly at its distal tip. J Neurosci 15:6827-6833.

Zheng J, Buxbaum RE, Heidemann SR (1993) Investigation of microtubule assembly and organization accompanying tension-induced neurite initiation. J Cell Sci 104:1239-1250. 